\title{
Change management and its effects on organizational resources
}

\author{
Roxana Mironescu, Ph.D., "Alecsandri" University of Bacau, Romania
}

\begin{abstract}
Change is a factor of strong impact especially on the managerial functions related to coordination and training of resources. Change Management has a broad spectrum of interpretations, referring to several types of changes, but is generally used in the management processes, in strategic management and the management of information, including the electronic function of information. Human Resources Change Management can be reactive or proactive, in the latter case the conditions for initiating changes that are part of the strategic objectives of the organization.
\end{abstract}

\section{Keywords:}

change management processes, resistance to change, performance, organizational resources.

\section{Change, as a condition to adapt the organization to the environment.}

Managing change relates to the adoption of change in a planned, structured and organized environment. In the middle of change management lies a set of questions like: how do we make this problem become more innovative, more competitive and more productive? What are the needed changes? What performance measures are we trying to adopt? Why people feel the need to be more creative? An important question relating to organizational change is "Why organizations change?", And the answer may be:

-for having poor performance;

-to adapt to changes from the external environment;

-to achieve or maintain a competitive advantage (better price, high quality) from the competition;

-to exploit an innovation.

We can further say that there are two sources that determine organizational change: - external sources - the change is initiated or imposed by the actors, interest groups from outside the organization;

- internal sources - change starts from groups or individuals such as shareholders, management, employees.

Another fundamental question concerning the subject of change can be: "What can we change in organizations?" There are frequently changing the following:

-objectives and strategies;

-technology;

-human resources;

-organizational structure;

-environment.

Professional practice concerns the reaction, the response to the changes that the organization can not control or it can do it only to a limited extent, such as, for example, changes occurring in law, in social or political climate. The necessary expertise of change management is made of : methods, models, techniques and other 
tools taken from sociology, psychology, economics, industrial engineering, systems engineering, the study of individual and collective behavior.

The adoption of specific methods for managing change emerges as a difficult but necessary continuous process, despite all the difficulties encountered. An important task for the manager of a group is to determine the group members to agree on what is expected to do the group, how things must be done and till when.the group must work together .

An important function of management is to drive change, because the environment is in a constant change, and the competition appears to be intense. Increasingly more, management is oriented to stimulate change and encourage adaptation and innovation in order to improve products and services, to meet new pressures and demands.

Change is stressful.

Processes involving organizational change involve individual changes, too. In the middle of efforts, incertitudes and tensions implied by the change, it must be found a small pause to reflect that change may be a good thing, a factor driving the advance and progress. Change is, according to Paton and McCalman's "an ongoing process of confrontation, identification, assessment and action", in which its managers find themselves to have a significant role, both as promoters of critical mutations, and as individuals invested with authority and status of implementation any mechanism of the future organizational and ideological configurations.

Kurt Lewin believe that change is a process in dynamic equilibrium, in which various forces are pressing to change the parameters as set out in the formal organization, but at the same time, creates a strong resistance to any system transformation in its whole or in its partial components. The most important determinants of change may be the following: technological change, high rate of aging of the products, improving working conditions, the flood of new information. And resistance to change is induced by: obsolete mentalities, individual or collective mental blockages, disinterest, fear of the unknown and new, fear of possible failures, the low level of professionalism, destructuring jobs.

To act, the manager must have a good control over the critical situation. Undergoing a stage that involves diagnosis of situations becomes imminent. The main phases of diagnosis are:

- identify the type of problem, which involves finding all critical events that may cause change, action that can be done through the list of issues, ideas box of ideas etc. The complexity of problems related to the possibilities of their solutions, which are available for the organization, there are the proper factors affecting the implementation of changes;

- formulation of positive and negative symptoms that cause problems;

- the causes that generated the problem and the effects upon their facts or they may have effects for each type of problem. It is essential to understanding the nature of those factors which have caused the problem and generated the positive or negative effects. The causes may be direct, indirect, primary and secondary, the effects can be identified as the immediate, medium and long term.;

- specifying the ways problems can be solved and also the resources involved in this process. The main ways of action will lead to the decrease of the negative effects and they amplify the positive effects, with emphasis on resources needed and there are available for such actions;

- the estimated results that will be reached by achieving change. In this last phase we can estimate the expected measurable and quantifiable aspects of efficiency that will arise from solving the problems of change.

Organizational change process is often a complex one, accompanied by risk, uncertainty and disorder, it involves the browse of several stages, and often, the final results are not up to the expectations and even often put under question the efforts. 
Adverse reaction to change is determined by several factors, of which we can mention:

- the fear of losing the income - any change is likely to stir up fear about the loss of jobs or a considerable reduction of revenues;

- the fear of destroying interpersonal relationships - people feel they have a greater psychological comfort when they work with people that they know. Unwritten rules of a group have a large effect in mobilizing individuals in achieving objectives, and that is why the strong groups show a great opposition when managers seek a change likely to modify the group composition;

- the need to learn - change force people to change their mode of action. For them it becomes necessary to assimilate knowledge, methods and new procedures, which require additional training, with an additional intellectual effort;

- the fear of the unknown - the changes are accompanied by uncertainty over their purpose, which determine the individual / team / group / organization to be withheld;

-conservatism - low tolerance to change;

-lack of information;

-lack of understanding the changes benefits.

In order to accept the changes, theory suggests that management must consider the following issues:

-creating the trust for the individuals undergoing change;

-preliminary discussion on how the change will be achieved;

-direct implication in the process of change;

-ensure that the change is reasonable;

-avoiding the threats;

-proper timing of change.

There are many models of organizational change and reflecting the process stages, but one of the best known is that of Lewin in 1951, which suggests that any change or innovation can be understood through three successive stages: unfreeze, change or movement (turning) and regelation. Lewin believes that organizations are essentially stable in structure, and change is the process by which organizations move between different stable states. This model was criticized for its"static" view of organizations. Lippit (Organization Renewal, 1982) speaks about the organizational crisis phases: shock, defensive, awareness, adaptation and change. Elizabeth Kübler-Ross (1970) establishes similarities between management change model and people's reactions when they are suddenly informed that they are sick with incurable illness: denial, rebellion, bargaining, depression and finally acceptance.

Organizational transformation involves changes at three levels. The first level is the change of employees' attitudes and behaviors in the organization. The second level refers to the change management system, and the last level concerns the deep layers of the organization, belonging to its culture, i.e. in the systems of values, beliefs, affectivity of community.

Another feature of the organizational transformation is that change are not resuming to the simple maintenance of functional organizational system, but they aim the renewal of the organization as a whole.

Any change requires the various exploratory and consultation, a preliminary analysis and a plan for implementing change. It is very important in a change approach is the employment of a specialist / consultant from outside the organization.

Change can be approached in a variety of ways. There is not an optimal strategy in all circumstances, sometimes it is useful the combination of strategies for successful change process. Next we describe several strategies that can be used in the process of change. The approach was made according to the degree to which it is imposed to the change subjects: 
- dirijiste strategy: the right allowed to managers to manage change. When this approach is used, managers are claimed to use their authority to impose change. The advantage of this strategy is that change can be implemented very quickly, as required, involving a small number of people. The disadvantage is that it takes not account of the views or feelings of those affected by change, thereby losing the valuable information, information that can lead to lower implementation rate of change, or even undermine them;

- strategies based on expert: change management as a way of solving a problem. This approach is applied when the change results from a technical problem whose solution requires a solution given by experts (eg introducing a new information system). Such a change is implemented by special project teams - with precise instructions and guidance from managers, with little involvement from those who bear the change consequences. The advantage:the use of the experts' knowledge, it requires a relatively small group, the implementation is relatively fast. Disadvantages: change subjects might have different oppinions face the experts and they do not accept the legitimacy of the solutions adopted by the experts. Therefore, resistance to change can occur;

- negotiating strategy: the enforcement of negotiations on the change. Approach supposes a desire to negotiate, accepting the idea that it would take some adjustment and concessions. All this do not absolve the responsible managers, but recognizes that people affected by the change are entitled to express their oppinion on this regard, or they have the power to resist to changes (if not persuaded to join it). The advantage of this approach is that the change subjects have the opportunity to make public their oppinion, so they will be less likely to resist. The disadvantage is that implementation might take some more time, and the results are provided as easy as it is estimated;

- educational strategies: change management is to attract emotional and rationalist subjects. This approach involves changes in the system of values and beliefs of people, so that they are supportive for change and adhere to a common set of organizational values. The emphasis is on emotional and rational conquest by a combination of activities, such as persuasion, education, training and selection. The advantage of this approach is the involvement of people in implementing change (people are convinced of its necessity). The disadvantages are that this approach requires a longer period of time for implementation and resource consumption can be relatively large;

- participatory strategies: we are all involved in the change application. The subjects are involving in change (directly or through representatives), managers are determined to initiate change, the groups involved in carrying them out (less dominated by persons with management authority) and even some organizational development consultant (to facilitate the conduct this process). Advantages: more likely the change is accepted, people engage in persuasion for implementing change, a better chance of individual and organizational learning. Disadvantages: implementing change is relatively slow, change management effort is complex, it is likely to require more resources.

The five strategies described above have affinities with other aspects of management, such as power and influence strategies and leadership / management styles. When opting for a strategy we take into account not only the circumstances we face, but also the prefered managerial style.

Each strategy may be appropriate in different circumstances and the factors affecting the choice of the strategy there are:

-the urgency of the situation - as the threat is more pressing and more important,so the strategy is more rapidly chosen;

-the degree of the expected opposition - as the opposition is greater, the more appropriate can be a slower strategy which may overcome resistance to change; 
-the power-base of the change initiator - if the developer is strong enough, it is possible to apply a rapid change, even if there is a risk of a strong opposition;

-the need of information and responsible engagement -a rapid change means that the developer knows very well what it is necessary to be done, otherwise he would be forced to involve other persons and to advance more slowly.

Change management can be a stressful and demanding work. The way people relate to change can be affected by the position they have in the organization and the attitudes they adopt face to change affect the role that they assume most easily in the process of change, but also the opposite is valid :the attitudes can be shaped by the role played in the change process.

There are identified three different roles in the process of change:

- strategists and initiators of change, initiating and determining the direction of change;

- implementers of change, responsible for coordinating and implementing change;

- subjects of change, which are deeply influenced by the change and its implementation.

These roles are not mutually exclusive. Strategists may be involved in implementing change, the subjects, although they have less power control on change may have some influence in changing strategy or its implementation.

When we consider a change we should consider the following questions: "It is the change necessary ?" Is the change feasible?" and "What is the level of sustainability of the change process?"

It is possible that change must be essential, but it does not mean that every possible project must be put into practice. New and different things are not always better, and even if they are so, the costs of introducing changes may be too high.

Based on these considerations, it was conceived "Change Equation" theory as a tool to highlight what it should be done to initiate a change. The basis of this equation lies on the assumption that people are not interest in changebut rarely, if the supporting factors are not exceeding the costs of change.

The "Change Equation" is the following:

$$
\mathrm{A}+\mathrm{B}+\mathrm{C}>\mathrm{D} \text {, where: }
$$

$\mathrm{A}=$ level of dissatisfaction of the individual or of the group to the existing stage;

The dissatisfaction we refer to, may be not shared by other members of the team / group. If the others consider the existing status quo suits them, it is unlikely that they will support change.

$\mathrm{B}=$ individual or group view on a better future;

In order to support a change, individual or group in question must have a well defined and clear vision, on a better way of doing things, otherwise people will not try to implement it. There are several risks about the possible existence of several oppinions and then, people will scatter energy debating contradictory;

$\mathrm{C}=$ there is a first acceptable and risk free step ;

The first steps are acceptable if they are small and likely to succeed, or it is clear that in case of failure, the situation can be easily and without problems resolved ;

$\mathrm{D}=$ costs that the individual / group / organization have to bear;

The considered costs can be expressed in money, resources, time, energy and adverse psychological and personal effects. We suppose to be important the charged costs, not necessarily the real ones and what is trivial for some people, may prove to be stressful for others.

Consequently, the problem of change is, a dimension of content, but also, another dimension, the process one. Therefore, it is creating, at least, three theoretical and methodological approaches of change management, such as: its management in a reactive or proactive way, the existence of areas of expertise and professional practice (with the dependent variable skills and abilities of practitioners of change) 
and the confirmation of a methodological set of tools, consisting of models, techniques, methods and other instruments, able to accurately reveal the causes, effects and the manner to maintain a favorable change process climate.

\section{References}

1. Scullion, H, Linehan, M. (2005), International Human Resources Management, Palgrave Mac Millan, New York;

2. Bennis, W, Benne K.D., Holt, R. C.(1969), The Planning of Change, Rinehart and Winston, New York;

3. ***,(2002) Managementul resurselor umane, "Înţelegerea schimbării”, CODECS;

4. ***,(2002), Putere, conducere şi schimbare, "Managementul schimbării", CODECS;

3. http://en.wikipedia.org./wiki/Change Management. 